\title{
Data Fusion for Relative Localization of Wireless Mobile Nodes
}

\author{
Carmelo Di Franco, Gianluca Franchino and Mauro Marinoni \\ Scuola Superiore Sant'Anna, Pisa, Italy \\ Email: \{c.difranco,g.franchino,m.marinoni\}@sssup.it
}

\begin{abstract}
Monitoring teams of mobile nodes is becoming crucial in a growing number of activities. When it is not possible to use fix references or external measurements, a practicable solution is to derive relative positions from local communication. In this work, we propose an anchor-free Received Signal Strength Indicator (RSSI) method aimed at small multi-robot teams. Information from Inertial Measurement Unit (IMU) mounted on the nodes and processed with a Kalman Filter are used to estimate the robot dynamics, thus increasing the quality of RSSI measurements. A Multidimensional Scaling algorithm is then used to compute the network topology from improved RSSI data provided by all nodes. A set of experiments performed on data acquired from a real scenario show the improvements over RSSI-only localization methods. With respect to previous work only an extra IMU is required, and no constraints are imposed on its placement, like with camera-based approaches. Moreover, no a-priori knowledge of the environment is required and no fixed anchor nodes are needed.
\end{abstract}

\section{INTRODUCTION}

Teams of mobile nodes are effective solutions for a broad range of applications. For instance, a team of robotic nodes can be used to accomplish several tasks, such as cleaning of hazardous area, surveillance, monitoring, exploration, search and rescue, and transportation. In one hand, a robotic team can avoid the human presence in risky situations, in the other hand it can represent the most convenient solution in terms of performance, costs, and efficiency. Another example regards localization systems used to track and support human workers operating in dangerous areas [1].

The coordination of a set of mobile nodes requires to solve different problems, such as nodes localization, data communication, and tasks allocation. This paper is focused on the localization problem, which is of paramount importance to guarantee the cooperation among the team members. In some situations, it might be possible to exploit an infrastructure that makes feasible to derive the absolute positions. An example of such as an infrastructure is the Global Positioning System (GPS) which is a very common solution; nevertheless, it is not a feasible solution in indoor environments or in particular places where the signal is not available. An alternative solution is to build a dedicated infrastructure,which is often costly and not feasible in emergency situations.

In common situations, nodes frequently transmit messages to collaborate. Thus, inter-nodes distances can be measured from radio signals exploiting different techniques. In particular, a node can extract Received Signal Strength Indicator (RSSI) information from any received packet to derive the distance from the packet source. Therefore, any exchanged packet can be used to determine the relative distance of each node. However, RSSI information is affected by multi-path fading phenomenons and quantization noise that can cause large errors on distance estimation. Besides RSSI methods, the distance between wireless nodes can be computed by using techniques based on signal propagation delays. Some of them require global time synchronization, as Time of Arrival (ToA) [2] [3] and Time Difference of Arrival (TDoA) [4] [5] measurements. Others, like Round-Trip Time-of-Flight [6] [7], now on Time of Flight (ToF) for brevity, do not need global time synchronization, since based on unilateral measurements. Alternative approaches exploit Angle of Arrival (AoA) techniques that need antenna arrays to work [8] [9].

In addition to radio signals, the position of a mobile unit can be estimated by IMU sensors. An IMU is a device usually composed by accelerometers, gyroscopes and, sometimes, a magnetometer. By an IMU it is possible to measure velocity, orientation, and gravitational forces on a body. Hence, a mobile robot equipped with an IMU can derive its speed and position integrating the acceleration with respect to time. Unfortunately, the acceleration values provided by an IMU suffer a small bias that, together with the integration process, causes drift errors to the position measures.

To increase the accuracy of the measured distances, an effective solution is to combine different sources of data.

\section{A. Contributions and summary}

This work proposes a localization method that combines inertial data provided by IMU sensors, ToF, and RSSI information obtained from radio communications, to derive internode distances. These distances are stored by each node in a distance matrix used to compute the relative coordinates of every team member through a weighted Multidimensional Scaling (wMDS) algorithm [10].

The major advantage of the proposed solution is that the precision of distance measurements is improved by combining IMU and ToF with RSSI information, maintaining the fast dynamics of RSSI readings. Moreover the proposed system does not use expensive hardware, since IMU sensors and radio transceivers that provide RSSI information are widely used and not expensive. Moreover, nowadays there are manufacturers that provide low cost devices for ToF measurements [11].

The rest of the paper is organized as follows. Section II analyzes the related work, Section III describes the localiza- 
tion system in detail, Section IV shows the simulation and the experimental results and, finally, Section VI states out conclusions and future work.

\section{RELATED WORK}

A lot of research has been carried out on the problem of indoor localization. Several solutions are based on timebased techniques, RSSI techniques, and some others exploit IMU sensors. More recent works proposed hybrid methods that combine different types of data, e.g RSSI and IMU data, to improve the accuracy of position measures. The main techniques used for data fusion are based on Kalman [12], Bayesian and Particle [13] filtering.

Oliveira et al. [14] proposed an anchor-free localization algorithm intended for small multi-robot teams. By combining both the ToF and RSSI ranging, the algorithm performs an online estimation of the indoor log-distance path loss model of the radio channel. This model is then used, together with an Extended Kalman Filter (EKF) [12], to track the distance between every pair of units. In [15], the authors proposed another approach to fuse RSSI and ToF information that, differently from [14], assumes the channel parameters to be estimated in advance.

Some other research works base their localization systems on both IMU devices and Ultra-WideBand (UWB) radio positioning technology, which is mainly based on TDoA and AoA techniques. Corrales et al. [16] proposed a hybrid tracking system for the localization of a person in a workplace. The system is composed by an inertial motion capture system, used to track the movements of the person's limbs, and by an UWB localization system. The positions measured by both systems are combined through a Kalman Filter (KF) to improve the measurements accuracy. Benini et al. [17] presented an indoor localization system for mobile agents combining data provided by a commercial UWB localization system and a low-cost IMU by means of an EKF. In [18] the same authors extended their method by adding position data coming from a visual odometry system based on markers. The proposed solution is utilized to track the position of a small Unmanned Air Vehicle (UAV). Savioli et al. [19] proposed an indoor localization system that combines position data coming from an UWB localization system and inertial sensors. The data fusion is obtained by a fixed-gain steady KF that lowers the computational complexity of the algorithm, allowing its implementation on resource limited devices, typically used in wireless sensors network applications.

Some other works proposed methods to combine RSSI and IMU data. Malyavej et al. [20] considered the localization problem of indoor mobile robots. They proposed a localization method based on the fusion of RSSI data, coming from WiFi access points, and data coming from onboard IMU sensors. The sensor data fusion is obtained by an EKF. Schmid et al. [21] presented an experimental study on the pedestrian localization problem, which analyzes the improvements that can be obtained by fusing inertial data and RSSI data. This work compared the accuracy of a RSSI-only localization approach with respect to the RSSI and IMU data fusion approach. The authors concluded that the improvements obtained by fusing IMU and RSSI information reduces the positioning error to a certain amount, but the resulting accuracy is not significantly improved. However, they also concluded that the achievable localization accuracies suffices for the person localization scenarios considered in the experiments. Woodman and Harle [22] described a tracking system for pedestrian localization inside buildings. The proposed system uses a model of the building, a foot mounted IMU and a particle filter to deal with the typical drift problems of inertial sensors. The initial position of the tracked person is obtained by exploiting RSSI data obtained from a set of WiFi access points. The system is able to track the position of a person in buildings with multiple floors and stairs. The experimental results showed that, by using an ultrasonic localization system as ground truth, the resulting position accuracy is 0.5 meters for $75 \%$ of the time, and 0.73 meters for $95 \%$ of the time. Fink et al. [1] presented a localization system based on sensor fusion of RSSI data and positioning data obtained by an Inertial Navigation System (INS) composed by two accelerometers and one gyroscopic sensor. The precision of distance measures obtained by RSSI data was increased by adopting a diversity scheme based on antenna and frequency diversity and a KF to estimate and correct the drift errors of the INS. The proposed system has been evaluated in a testbed composed by eight anchor nodes, called reference nodes, evenly distributed in an overground longwall mining. The node to be tracked is carried by a person that moves linearly among the reference nodes. The experiments highlighted that the localization system shows an average estimation error of 1.68 metres. Li et al. [23] presented a method to track mobile nodes that fuses WiFi RSSI data and inertial data from a smartphone. The proposed solution is composed by a Sequential Monte Carlo Kalman Filter (SMC-KF), which elaborates the navigation data coming from the smartphone IMU, and a Steepest Descent Random Start (SDRS) algorithm that elaborates the RSSI data. The performance of the proposed approach has been assessed by simulation experiments an compared with solutions based on IMU data elaborated by an EKF, and solutions based on UWB radio-location devices.

In the solutions reported above, RSSI and UWB positioning data are gathered exploiting a set of wireless nodes used as reference anchors. The work proposed in this paper does not rely on fixed anchors, but the RSSI data are gathered from the local communication among the robotic team members. Note that, the main advantage of an anchor-free approach is that it does not require a fixed infrastructure, making the system more flexible and cheaper.

\section{SySTEM DESCRIPTION}

The system is composed by $n$ mobile nodes $\left\{\eta_{1}, \ldots, \eta_{n}\right\}$ that communicate through a radio channel forming a fully connected network. Each node is equipped with an IMU and a radio system that provides both RSSI and ToF information. 
The localization is performed by two sub-systems. A first one, called Distance Measurement Sub-system (DMS), which estimates the inter-nodes distance every time a packet is received, and a second sub-system, which use these inter-node distances to compute the relative positions of all nodes.

Each node $\eta_{i}$ implements the DMS, which estimates the inter-node distances from RSSI, ToF, and IMU data collected by the node. Each node estimates its speed using data acquired from the onboard IMU.

When a new ToF information is available it is possible to estimate the channel model. We use the RSSI value to estimate the distance every time a packet is received. It is also important to note that each node sends its estimated speed inside the packet: when a node receives a packet it can use the information regarding the transmitter speed and its own estimated speed to estimate their relative speed and improve the accuracy of the estimated distance with the RSSI.

In particular, each DMS $i$ is composed by four main blocks, as shown in Figure 1:

- A filter that mitigates the noise and the quantization errors present in the RSSI data implemented as a median sliding window;

- An on-line channel estimator based on ToF information, used to characterize the relation between the nodes distance and the RSSI;

- A KF that estimates the nodes movements from IMU data;

- An EKF that integrates the output of the other blocks and provides a better estimation of the distance between nodes.

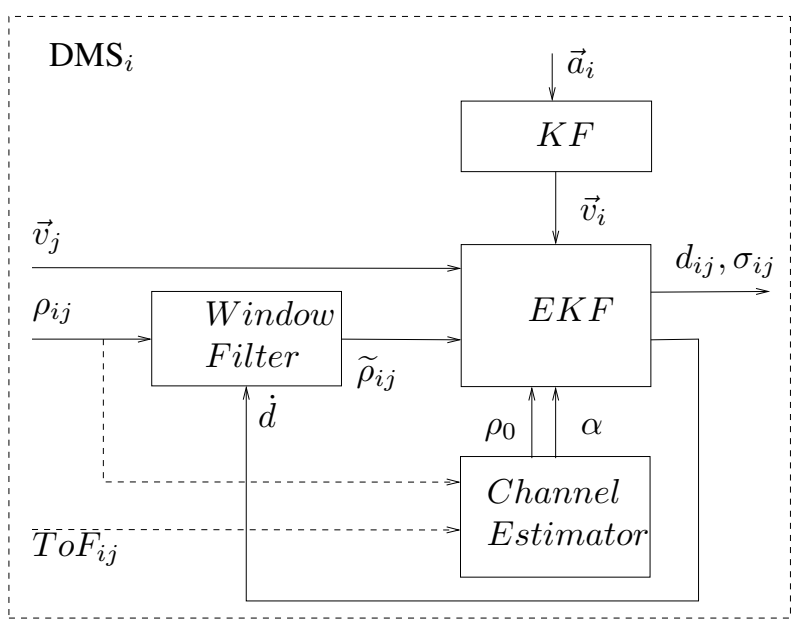

Fig. 1. Overview of the inter-robot Distance Measurement Sub-system (DMS) on node $\eta_{i}$.

Figure 1 shows the block diagram that illustrates the whole sub-system. Each packed received from node $\eta_{j}$ provides information regarding the node speed $\vec{v}_{j}$ and $\rho_{i j}$ that is the RSSI of the packet. The $\mathrm{ToF}_{i j}$ value provided by the ToF technique is used to estimate the channel model. $\vec{a}_{i}$ is the acceleration vector from the node's IMU. The EKF estimates the relative speed between $\eta_{i}$ and $\eta_{j}$ used to dynamically control the median sliding window filter as described in detail in Section III-B. The sub-system $D M S_{i}$ produces two outputs: an estimation of the distance $\left(d_{i j}\right)$ between $\eta_{i}$ and $\eta_{j}$ and its covariance $\sigma_{i j}$. The approach shown in Figure 1 not only gives better estimation of distances, but also provides information regarding the quality of each distance depending on the actual noise level and node mobility.

All these data are collected and integrated in the second subsystem to compute the map with all the nodes relative locations. A technique widely used to perform the estimation is the Multidimensional Scaling (MDS) [24], which is a method that represents measurements of similarity (or dissimilarity) among pairs of objects as distances between points in a N-dimensional space. Applications of the MDS algorithm include scientific visualization and data mining in several fields [24], including it also can be used to find a relative map between nodes [25]. The algorithm takes as input data pairwise (dis)similarities (e.g., distances) and returns a set of coordinates as a relative map. Given $n$ nodes in two dimensions and the estimated pairwise distances $d_{i j}$, MDS recovers the nodes coordinates $\vec{x}_{i}$ for all nodes minimizing the mismatch between the estimated distances $\widehat{d_{i j}}$ and the distances $d_{i j}\left(\vec{x}_{i}, \vec{x}_{j}\right)$ corresponding to the unknown coordinates $\vec{x}_{i}$. The mismatch is called Stress function. If a reliability information is associated with the measured distance, it is possible to use wMDS [10]. In wMDS, the Stress function is defined as:

$$
\begin{aligned}
S\left(\vec{x}_{1}, \ldots, \vec{x}_{n}\right) & =\sum_{i=1}^{n} \sum_{j \neq i, j=1}^{n} w_{i j}\left[\widehat{d_{i j}}-d_{i j}\left(\vec{x}_{i}, \vec{x}_{j}\right)\right]^{2}+ \\
& +\sum_{i=1}^{n}\left\|\vec{x}_{i}-\widehat{\vec{x}_{i}}\right\|_{2}^{2}
\end{aligned}
$$

where $w_{i j}$ is the weight associated with the corresponding distance. In our system it is possible to define the weight as a function of the covariance information from the output of the EKF of the first sub-system. As shown in Figure 2, each node $\eta_{i}$ estimates all the distances $d_{i j}$ and the covariance $\sigma_{i j}$ for the nodes $\eta_{j}$ with $D M S_{i}$. This information is aggregated inside the block Collector which produces the distance matrix $D$ and the weight matrix $W . D$ is a $n \times n$ matrix where each item $i, j$ corresponds to the estimated distance $d_{i j}$ (on the diagonal $d_{i i}=0$ ). Since wMDS requires a symmetric matrix, each item $D_{i j}$ of the matrix is calculated as the mean between $d_{i j}$ and $d_{j i} . W$ is a $n \times n$ matrix where each item is obtained as a function of the covariances $\sigma_{i j}$, as better described in Section III-D. wMDS also receives as input a set of coordinates used as a starting point. Using set of estimated coordinates computed in the previous iteration as an input for the next wMDS estimation, it is possible to reduce the error in the map topology of the nodes, as will be showed in detail in Section V. The wMDS algorithm can be distributed on each node, or executed on one of them, or on a central station. The estimated distances by the nodes are exchanged as part of the wireless communication, to aggregate the matrices and execute the wMDS algorithm in one of the tree ways mentioned above. 


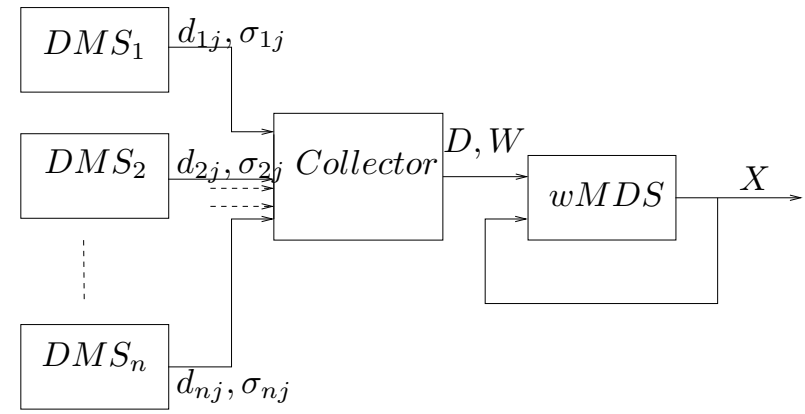

Fig. 2. Overview of the sub-system than computes the relative position of each robot.

\section{A. Online channel estimation using ToF}

$\mathrm{RF}$ power decays as the electro-magnetic waves travel through air. By measuring the RSSI of a message, and using a propagation model, it is possible to infer the distance to the transmitter. However, such a computation requires the knowledge of several parameters, as the transmission power, antenna gains, frequency of the carrier, and medium characteristics. In open space, the relationship between signal strength and distance can be represented by the log-distance path loss model [26]:

$$
\rho_{d}=\rho_{0}-10 \alpha \log \left(\frac{d}{d_{0}}\right) \Longleftrightarrow d=d_{0} \times 10^{\left(\rho_{0}-\rho_{d}\right) /(10 \alpha)}
$$

where $\rho_{d}$ is the RSSI value at distance $\mathrm{d} ; \rho_{0}$ is the RSSI value at a reference distance $d_{0}$ (we consider $d_{0}=1$ ), which includes the aggregated effects of transmission power, antenna gains, and frequency attenuation; and $\alpha$ is the path loss exponent that represents the propagation medium properties.

When a node sends a packet, the receiver obtains the RSSI from the transceiver and uses Equation (2) to compute the distance. However, a proper propagation model requires the estimation of the equation parameters, namely the reference RSSI value $\rho_{0}$ at the respective reference distance $d_{0}$, and the path loss exponent $\alpha$. In this work, the distance obtained with the ToF technique is used to estimate the channel model: we define a vector $g$ of predefined $m \log$-separated distances and create the matrices $A$ (size $[m+1,2]$ ) and a vector $b$ (size $m+1)$, considering $d_{0}=1$.

$$
A^{k}=\left[\begin{array}{cc}
1 & -10 \log (g(1)) \\
1 & -10 \log (g(2)) \\
\vdots & \vdots \\
1 & -10 \log (g(m)) \\
1 & -10 \log \widehat{d^{k}}
\end{array}\right], b^{k}=\left[\begin{array}{c}
\rho_{0}^{k-1}-10 \alpha^{k-1} \log (g(1)) \\
\rho_{0}^{k-1}-10 \alpha^{k-1} \log (g(2)) \\
\vdots \\
\rho_{0}^{k-1}-10 \alpha^{k-1} \log (g(m)) \\
\widehat{\rho^{k}}
\end{array}\right]
$$

The first $m$ lines represent the previously estimated model $\widehat{X^{k-1}}$, while the last element represents the new measurement. Then, the new channel model $\widehat{X^{k}}$ is obtained using the Maximum Likelihood Estimator (MLE):

$$
\widehat{X^{k}}=\left[\begin{array}{c}
\widehat{\rho_{0}^{k}} \\
\alpha^{k}
\end{array}\right]=\left(A^{T} A\right)^{-1} A^{T} b .
$$

This allows to run the MLE algorithm using a fixed number of samples $(m+1)$, and to fuse the new knowledge into previous one, where $m$ defines the weight of the new measurement.

\section{B. Using inertial measurement to improve RSSI readings}

The velocity of a node $\eta_{i}$ can be used to improve the windowed RSSI filter previously described by re-sizing the length of the window accordingly to the velocity, which is estimated implementing a KF.

Let us define the input of the window filter for the link between nodes $\eta_{i}$ and $\eta_{j}$ at instant $k$ as:

$$
\Phi_{i j}^{k}=\left[\rho_{i j}^{k}, \rho_{i j}^{k-1}, \ldots, \rho_{i j}^{k-\varphi_{i j}^{k}}\right]
$$

where $\rho_{i j}^{k}$ is the Received Signal Strength of the packet transmitted from $\eta_{j}$ to $\eta_{i}$ at instant $k$, and $\varphi_{i j}^{k}$ is the window length at that time. We want to define a relationship between $\varphi_{i j}^{k}$ and the relative velocity between $\eta_{i}$ and $\eta_{j}$. If a node moves, the window filter will contain inside his window values of the RSSI measured at different positions. To avoid this inconsistency we impose that the difference between the distance at first instant in the time window $d_{i j}^{k}$ and the last one $d_{i j}^{k-\varphi_{i}^{k}}$ has to be smaller than the standard deviation of RSSI:

$$
\left|d_{i j}^{k}-d_{i j}^{k-\varphi_{i j}^{k}}\right|<\delta_{R S S I} .
$$

Assuming a constant speed in the interval $\Delta t$, it is possible to express the relative speed as:

$$
\left|\dot{d_{i j}}{ }^{k}\right|=\frac{\left|d_{i j}^{k}-d_{i j}^{k-1}\right|}{\Delta T}
$$

where $\Delta T$ is the interval between two consecutive received packets from $\eta_{j}$. Equations (5) and (6) can then be combined to compute a bound for the window size:

$$
\varphi_{i j}^{k}<\frac{\delta_{R S S I}}{\left|\dot{d_{i j}}{ }^{k}\right| \Delta T} .
$$

Equation (7) can be showed in a graphical view as a hyperbolic curve (Fig. 3). In order to maintain the window size $\varphi_{i j}^{k}$ constrained between a higher and a lower bound, Equation (7) has been modified as follows:

$$
\begin{array}{r}
\varphi_{i j}^{k}<\frac{\delta_{R S S I}}{\left(\gamma \cdot \dot{d_{i j}}{ }^{k}+\dot{d}_{0}\right) \Delta T}+\varphi_{\min }, \\
\dot{d}_{0}=\frac{\delta_{R S S I}}{\varphi_{\max } \Delta T}
\end{array}
$$

where $\dot{d}_{0}$ is needed to return a finite value when the node is in steady state, and $\gamma$ is a constant proportional to the maximum acceleration of the system. Thus, $\varphi_{\max }$ and $\varphi_{\min }$ define the maximum and the minimum size of the window, and $\gamma$ increases or reduces the slope of the curve. Since the size of the window must be a natural number, the function is approximated with its ceiling, thus $\varphi_{i j}^{k}$ results in a step function, as shown in (Figure 3). 


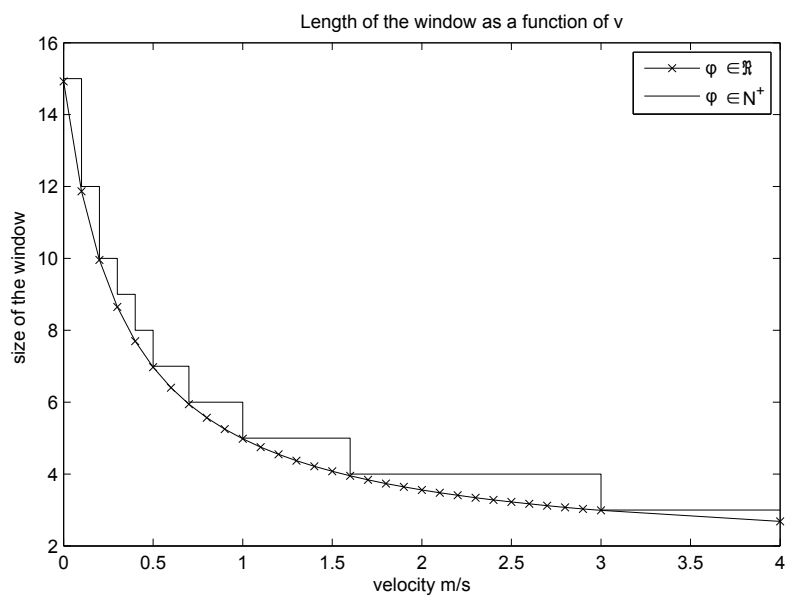

Fig. 3. Size of the median sliding window filter as a function of the speed.

\section{Using IMU and KF to estimate the node speed}

As typically used in robotics, movements tracking is performed by IMU sensors. To estimate the node speed $\overrightarrow{\dot{x}}$ and the acceleration $\overrightarrow{\ddot{x}}$, a KF as been implemented. The State equation at time $t=s \cdot \Delta t$ is defined as follow:

$$
\begin{gathered}
X^{s}=\left[\begin{array}{cc}
1 & \Delta t \\
0 & 1
\end{array}\right] X^{s-1}+\left[\begin{array}{cc}
\frac{\Delta t^{2}}{2} & 1 \\
0 & \Delta t
\end{array}\right] \psi^{s} \\
X=\left[\begin{array}{ll}
\overrightarrow{\dot{x}} & \overrightarrow{\ddot{x}}
\end{array}\right]
\end{gathered}
$$

where the state vector $X$ is defined in Equation (11) and $\psi^{s}$ is the Gaussian noise of the state. Since accelerations acquired by IMU are the only input measurement, the measurement vector $Z$ will be in this case a scalar. The measurement equation is defined as:

$$
Z^{s}=\left[\begin{array}{ll}
0 & 1
\end{array}\right] X^{s}+\nu^{s}
$$

where $\nu^{s}$ is the Gaussian noise of the measurement.

\section{IMU and EKF for Distance Tracking}

When node $\eta_{i}$ receives a packet for $\eta_{j}$, it also receives its estimated speed. Node $\eta_{i}$ can use this information to track the relative distance $d_{i j}$. This information improves the distance tracking and is used as input for the median sliding window filter described in Section III-B. An EKF is used to fuse the RSSI readings with the estimated speeds to track the distance between the two communicating nodes. The state equation at time $t=k \cdot \Delta T$ is defined as:

$$
X_{i j}^{k}=\left[\begin{array}{c}
d_{i j}^{k} \\
\dot{d_{i j}^{k}} \\
{\overrightarrow{v_{j}}}^{k} \\
{\overrightarrow{v_{i}}}^{k}
\end{array}\right]=\left[\begin{array}{c}
d_{i j}^{k-1}+\dot{d}_{i j}^{k} * \Delta T \\
\left\|{\overrightarrow{v_{j}}}^{k-1}+{\overrightarrow{v_{i}}}^{k-1}\right\| \\
{\overrightarrow{v_{j}}}^{k-1} \\
{\overrightarrow{v_{i}}}^{k-1}
\end{array}\right]+\psi^{k}
$$

where $d_{i j}$ is the estimated distance between $\eta_{i}$ and $\eta_{j}$ and $\dot{d_{i j}}$ the corresponding estimated relative speed, $\overrightarrow{v_{i}}$ and $\overrightarrow{v_{j}}$ are the estimated speeds of the two nodes, and $\psi^{k}$ the state noise. The measure equation is:

$$
Z_{i j}^{k}=\left[\begin{array}{c}
\bar{\rho}_{d_{i j}}^{k} \\
\overrightarrow{\vec{v}}_{j}^{k} \\
{\overrightarrow{v_{i}}}_{k}^{k}
\end{array}\right]=\left[\begin{array}{c}
\rho_{0}-10 \alpha \log _{10}\left(d_{i j}^{k}\right) \\
{\overrightarrow{v_{j}}}^{k} \\
{\overrightarrow{v_{i}}}^{k}
\end{array}\right]+\nu^{k}
$$

where $\bar{\rho}_{d_{i j}}$ is the measured RSSI, $\bar{v}_{j}$ is the estimated speed of node $\eta_{j}$ received inside the packet, and $\bar{v}_{i}$ is the speed of $\eta_{i}$ estimated with its own Kalman filter, as described in Section III-C.

The EKF provides also a covariance matrix $P^{k}$ that gives a confidence regarding the accuracy of the measurements. The first element of the matrix $P^{k}$ denotes the confidence on the estimated distance $d_{i j}^{k}\left(\sigma_{i j}=P^{k}[1,1]\right)$ and is provided to the wMDS algorithm to be used as weight $w_{i j}^{k}$, computed as follows:

$$
w_{i j}^{k}=\min \left(1, \frac{\sigma_{m i n}}{\sigma_{i j}}\right)
$$

where $\sigma_{\min }$ is a constant representing the minimum covariance above which the measurement is considered trustable. This selection is in accordance to the wMDS algorithm, which requires $w_{i j}^{k} \in[0,1]$, where 1 represents a precise measurement while 0 an untrustable one.

\section{Simulation Results}

This section presents a simulation study carried out to test and validate the localization system proposed in this work. In the following experiments we are interested in improving the accuracy of the estimated distance and not in the channel model dynamical estimation [14]. Thus, we could assume without loss of generality that the simulation occurs under the hypothesis of the accurate estimation of the channel model.

\section{A. Simulation Setup}

Our methodology has been tested through a set of simulation experiments on MATLAB ${ }^{\circledR}$. An indoor environment has been simulated with standard channel model parameters $\rho_{0}=-38 \mathrm{dbm}$ and $\alpha=2$. In order to perform the simulations, we created a dataset of synthetic measurements based on previously collected data. In particular, the RSSI has been represented as a Gaussian noise with zero mean and standard deviation $\sigma_{r s s i}=4.43 \mathrm{~m}$. The ToF measurement, previously measured in [14], has been represented as a Gaussian noise with mean $\mu_{T o F}=-0.38 \mathrm{~m}$ and standard deviation $\sigma_{T o F}=0.60 \mathrm{~m}$, and the accelerometer as a Gaussian noise with $\mu_{i m u}=-0.1 \mathrm{~m} / \mathrm{s}^{2}$ and standard deviation $\sigma_{i m u}=-0.2 \mathrm{~m} / \mathrm{s}^{2}$. The parameters for the sliding window median filter have been defined as follow: $\varphi_{\max }=15$, $\varphi_{\min }=3$, and $\gamma=1$.

\section{B. Results}

The first simulation experiment was aimed at showing how the dynamic adaptation of the window size improves the accuracy of the distance. Two nodes $\eta_{1}$ and $\eta_{2}$ were placed at a starting distance $d_{12}^{0}=4 \mathrm{~m}$. Then node $\eta_{1}$ was moved with a given speed (about $1.3 \mathrm{~m} / \mathrm{s}$ ) forward and backward. 
To keep a common consistent information about the topology of the team, each node periodically sends a packet with its local distance matrix $D$, the weight matrix $W$ relative to the distance and the speed estimated from its EKF. Note that a packet is sent with a period $\Delta T=1 \mathrm{~s}$, while the accelerometer is sampled with a period $\Delta t=50 \mathrm{~ms}$. Figure 4 shows the real distance (dashed line) was compared with the distance estimated with RSSI-only (' $*$ ' marker), the dynamic window filter (solid line), and the EKF filter ('o' marker).

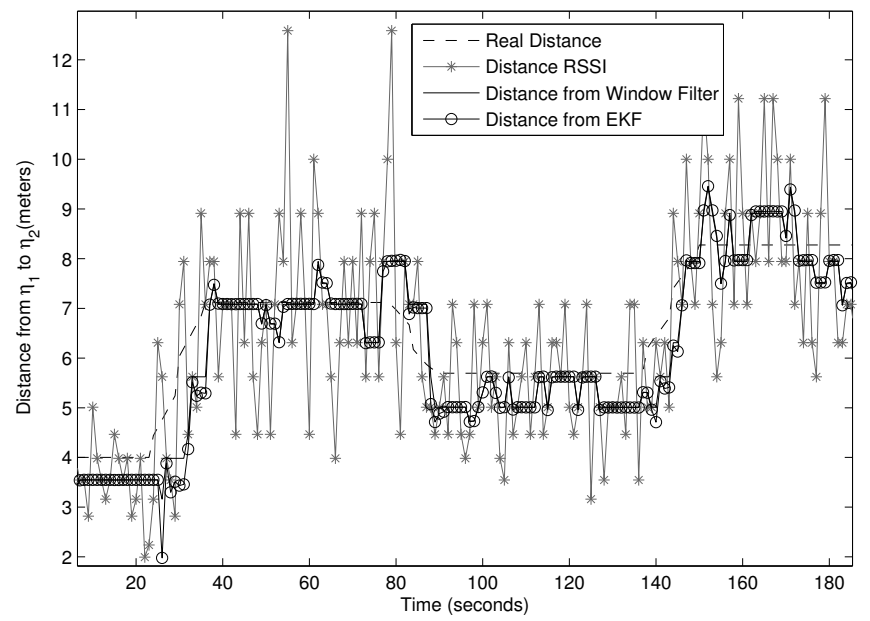

Fig. 4. Comparison between the real distance and the distance estimated with RSSI-only, the window filter, and the EKF.

Note that with RSSI-only the estimation presents large fluctuations due to the noisy measurements of RSSI, whereas using the window filter (that makes use of IMUs for controlling the filtering Window and the EKF) the spikes are dramatically reduced while the EKF estimates the relative speed and perform a feedback control on the window size.

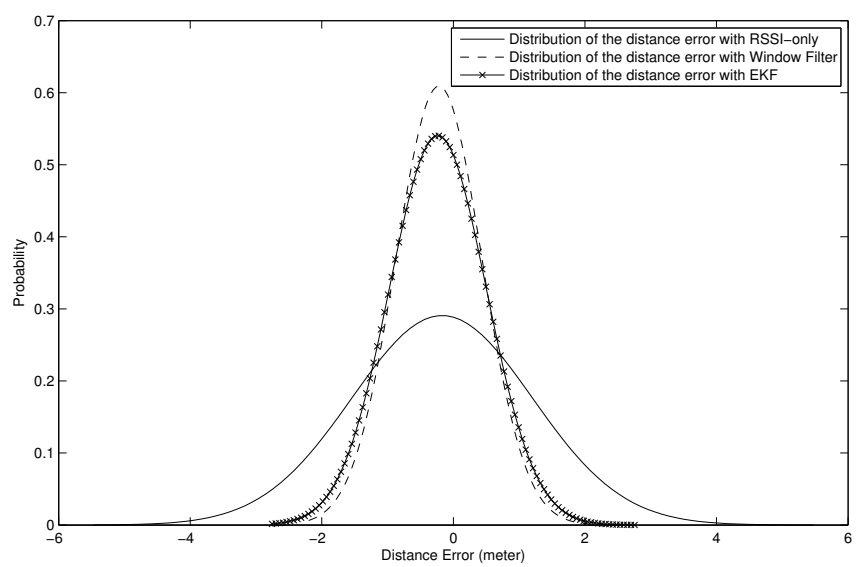

Fig. 5. Distribution of the mean error of the distance with RSSI-only, the window filter, and the EKF.

Figure 5 shows the distribution of the error between the real distance and the distance estimated with RSSI-only, the window filter, and the EKF. As expected, RSSI-only has a large standard deviation, while the window-filter and the
EKF have similar behaviors. The EKF use the RSSI data to block the drift on the speed caused by the integration of the accelerometer. Due to this motivation, our EKF follows more the RSSI than the velocity measurements. The window filter is more responsive because its window is updated dynamically by the EKF. However, it is worth noting that, even if the error is slightly worse, the EKF also provides the covariance matrix $P$ that is a confidence on how much we can trust the results. This information can be used as weight for the wMDS algorithm as described in Section III-D. Having a dynamic window size allows improving the accuracy when the nodes are stopped while permitting a fast dynamic. Figure 6a shows how the window filter adapts his size as a function of the estimated relative speed that is also compared with the real speed (shown in Figure 6b).
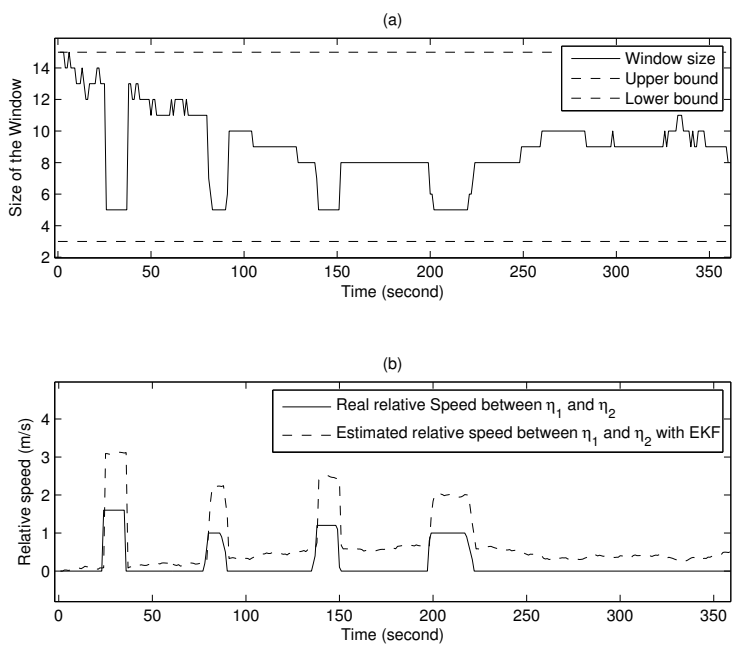

Fig. 6. a) How the window size adapts as a function of the estimated speed. b) Real speed compared with the estimated derivative of the distance.

To see how the size of the window filter affects the distance measurements, a simulation comparing dynamic window against two fixed windows of size 3 and 15 has been executed. Figure 7 shows the three estimated distances compared with the measured distance.

With a fixed window size $\varphi=3$, the estimation of the distance is noisy but follows the dynamics of the system. With a fixed window size $\varphi=15$, the estimation is much better, but of course the estimation has a higher delay that may be unacceptable if a node moves fast. In this cases, a solution based on a dynamic window can reach a higher precision in the estimation while supporting faster dynamics. This can easily be seen in Figure 8, where the error distribution of the three different implementations is showed.

\section{EXPERIMENTAL RESULTS}

This section presents a set of experimental tests performed to verify the feasibility of the localization method on real testbed. The experimental setup is composed by 4 Flex Mini 


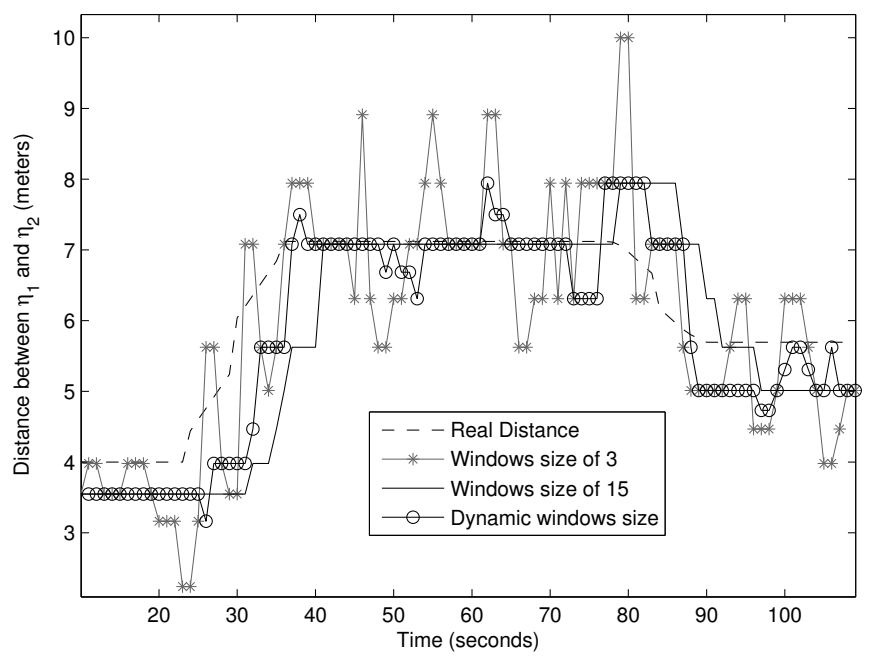

Fig. 7. Comparison between three simulation with a window size of 3,15 , and dynamic windows.

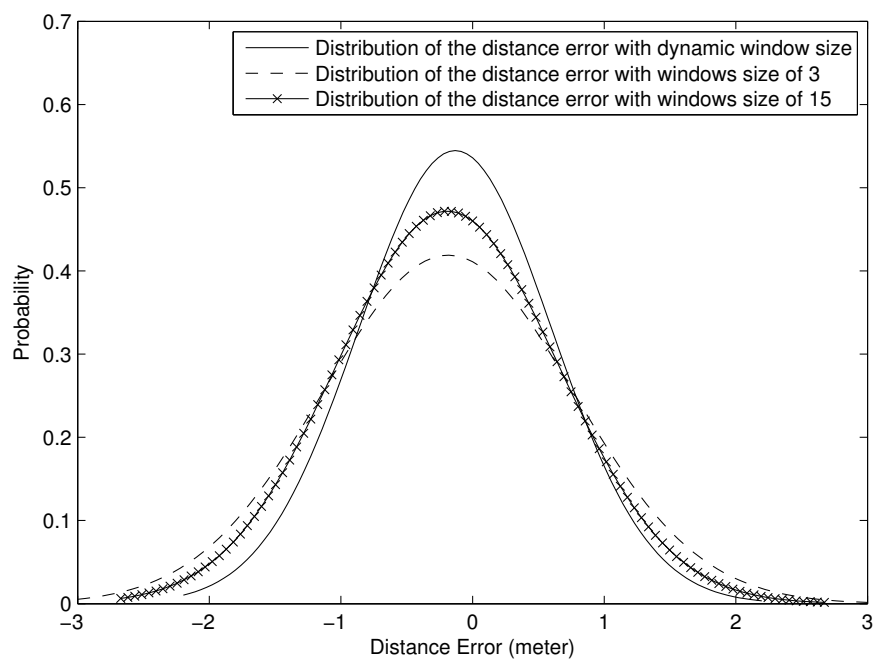

Fig. 8. Distribution of the distance error with window size of 3,15, and dynamic window

boards [27], each equipped with a 16-bits microcontroller and a radio transceiver compliant with the IEEE 802.15.4 standard [28]. The transceiver provides the RSSI of each received packet, represented by a 8 bits value. Each board is also equipped with an analog 3-axis accelerometer sampled by a 12-bit Analog to Digital Converter (ADC) of the board's microcontroller. Note that in this setup accelerations are the only data provided by the IMU.

\section{A. Results}

An experiment was carried out with 4 nodes at fixed positions to show how much the use of the wMDS algorithm improves the results in the relative map topology. Each $300 \mathrm{~ms}$ a master node sent a request to a specific node that answers with a broadcast packet. Each packet contains the distances and covariances data together with the estimated speed $v_{i}$ from the KF running in the source node. These values were collected and provided to a PC running the wMDS algorithm, implemented in MATLAB ${ }^{\circledR}$. Figure 9 and Figure 10 plot the outputs from the classical MDS and wMDS algorithms, respectively.

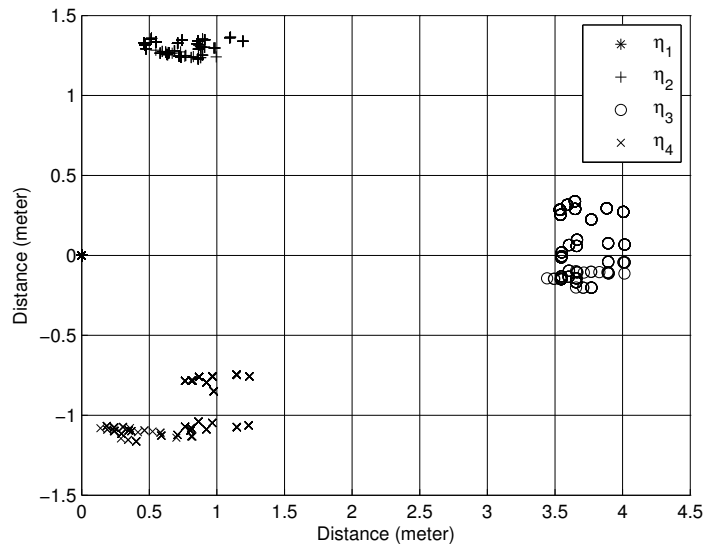

Fig. 9. Relative localization map computed using the classical MDS.

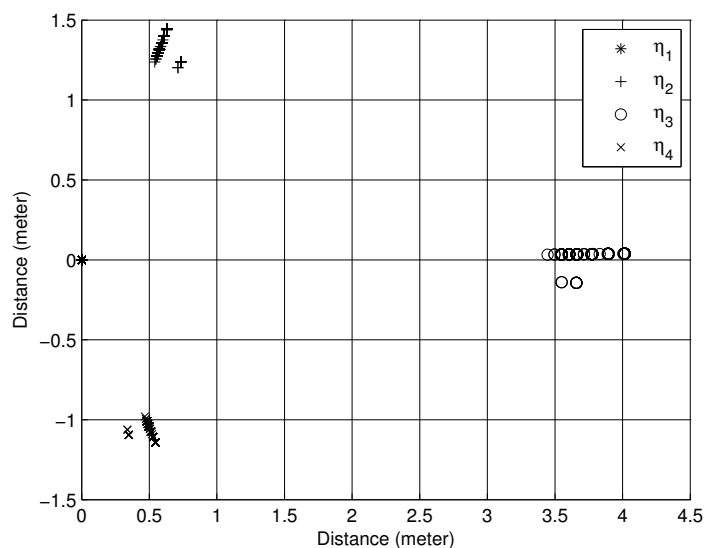

Fig. 10. Relative localization map computed using wMDS.

As clear from the plots, the wMDS (which uses the covariance matrix as a weight and the coordinates estimated at the previous time instant) exhibits better results. This is motivated by the fact that the knowledge of the topology at the previous time instant reduces the noise of the output map caused by the algorithm itself. Figure 11 shows the the error distribution of the difference between the measured distance and its mean, for all the nodes. The error distribution obtained with the wMDS presents a mean error $\mu=60.3 \mathrm{~mm}$ and a std. $\sigma=56 \mathrm{~mm}$ against the mean error $\mu=200.3 \mathrm{~mm}$ and the std $\sigma=112.1 \mathrm{~mm}$ of the classical MDS. It is important to highlights that this is not the error distribution of the distance (the correctness of the measured distance depends on the channel model estimation), but of the noise of the output by MDS.

\section{CONCLUSION AND FUTURE WORK}

This paper presented an anchor-free localization method intended for groups of mobile nodes. The proposed approach 


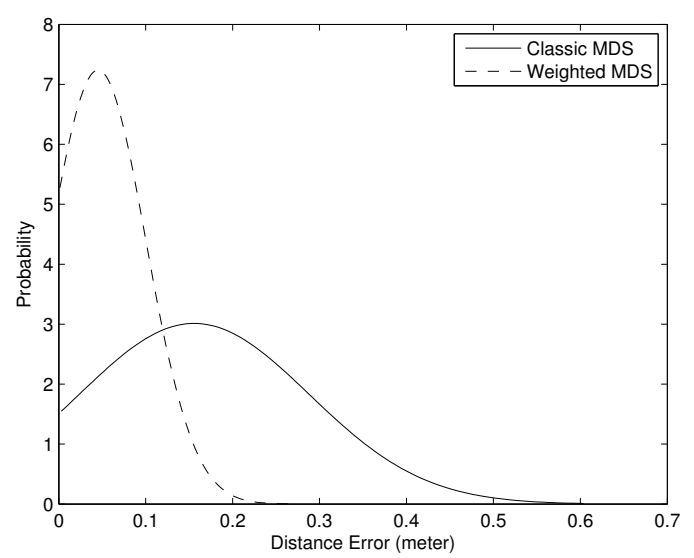

Fig. 11. Error distribution of the measured distance between node $\eta_{1}$ and the others nodes using MDS and wMDS.

exploits ToF and RSSI information, obtained from local communication, and IMU sensors to measure the relative distance between nodes. From the inter-node distances and by means of a wMDS algorithm, the localization system can derive for each node the relative position of the other group members. A set of simulation experiments, based on real data, has been carried out to show the accuracy of the proposed approach in comparison with RSSI-only localization methods. Moreover, the effectiveness of the proposed solution has been tested through an experimental evaluation.

In the future we want to improve the EKF with information from the wMDS output to further reduce the drift connected with IMU integration. In particular, we want to understand how the localization accuracy decreases as a function of the nodes speed and also intend to implement a distributed version of the wMDS to execute the whole localization algorithm on the team of nodes.

\section{REFERENCES}

[1] A. Fink, H. Beikirch, M. Vo, and C. Schrder, "RSSI-based Indoor Positioning using Diversity and Inertial Navigation," in Proceedings of the International Conference on Indoor Positioning and Indoor Navigation, IPIN 2010. IEEE, 2010.

[2] K. Cheung, H.-C. So, W.-K. Ma, and Y.-T. Chan, "Least squares algorithms for time-of-arrival-based mobile location," Signal Processing, IEEE Transactions on, vol. 52, no. 4, pp. 1121-1130, 2004.

[3] S. Gezici, Z. Tian, G. B. Giannakis, H. Kobayashi, A. F. Molisch, H. V. Poor, and Z. Sahinoglu, "Localization via ultra-wideband radios: a look at positioning aspects for future sensor networks," Signal Processing Magazine, IEEE, vol. 22, no. 4, pp. 70-84, 2005.

[4] N. B. Priyantha, A. Chakraborty, and H. Balakrishnan, "The cricket location-support system," in Proc. of the 6th international conference on Mobile computing and networking. ACM, 2000, pp. 32-43.

[5] F. Gustafsson and F. Gunnarsson, "Positioning using time-difference of arrival measurements," in Acoustics, Speech, and Signal Processing, 2003. Proceedings.(ICASSP'03). 2003 IEEE International Conference on, vol. 6. IEEE, 2003, pp. VI-553.

[6] T. E. Abrudan, A. Haghparast, and V. Koivunen, "Time synchronization and ranging in ofdm systems using time-reversal," IEEE Transaction on Instrumentation and Measurement, vol. 62, no. 12, 2013.

[7] B. Neuwinger, U. Witkowski, and U. Rückert, "Ad-hoc communication and localization system for mobile robots," in Advances in Robotics. Springer, 2009, pp. 220-229.
[8] D. Niculescu and B. Nath, "Ad hoc positioning system (APS) using AOA," in INFOCOM 2003. 22th Conference of the IEEE Computer and Communications. IEEE Societies, vol. 3. IEEE, 2003, pp. 1734-1743.

[9] P. Kułakowski, J. Vales-Alonso, E. Egea-López, W. Ludwin, and J. García-Haro, "Angle-of-arrival localization based on antenna arrays for wireless sensor networks," Computers \& Electrical Engineering, vol. 36, no. 6, pp. 1181-1186, 2010.

[10] J. A. Costa, N. Patwari, and A. O. Hero III, "Distributed weightedmultidimensional scaling for node localization in sensor networks," $A C M$ Transactions on Sensor Networks (TOSN), vol. 2, no. 1, pp. 39-64, 2006.

[11] Nanotron. (2010) nanoloc development kit. [Online]. Available: http://www.nanotron.com/EN/PR_nl_dev_kit.php

[12] S. Thrun, W. Burgard, D. Fox et al., Probabilistic robotics. MIT press Cambridge, 2005, vol. 1.

[13] J. V. Candy, Bayesian signal processing: Classical, modern and particle filtering methods. John Wiley \& Sons, 2011, vol. 54.

[14] L. Oliveira, C. Di Franco, T. E. Abrudan, and L. Almeida, "Fusing Timeof-Flight and Received Signal Strength for Adaptive Radio-Frequency Ranging," in International Conference on Advanced Robotics, 2013. ICAR 2013. IEEE, 2013.

[15] D. Macii, A. Colombo, P. Pivato, and D. Fontanelli, "A data fusion technique for wireless ranging performance improvement," Instrumentation and Measurement, IEEE Transactions on, vol. 62, no. 1, pp. 27-37, 2013.

[16] J. A. Corrales, F. A. Candelas, and F. Torres, "Hybrid tracking of human operators using IMU/UWB data fusion by a Kalman filter," in Proceedings of the 3rd ACM/IEEE International Conference on HumanRobot Interaction, (HRI) 2008. IEEE, 2008.

[17] A. Benini, A. Mancini, A. Marinelli, and S. Longhi, "A Biased Extended Kalman Filter for Indoor Localization of a Mobile Agent Using LowCost IMU and UWB Wireless Sensor Network Robot Control," in 10th IFAC Symposium on Robot Control. IFAC, 2012.

[18] A. Benini, A. Mancini, and S. Longhi, "An IMU/UWB/Vision-based Extended Kalman Filter for Mini-UAV Localization in Indoor Environment using 802.15.4a Wireless Sensor Network," Journal of Intelligent and Robotic Systems, vol. 70, no. 1, pp. 461-476, 2013.

[19] A. Savioli, E. Goldoni, P. Savazzi, and P. Gamba, "Low Complexity Indoor Localization in Wireless Sensor Networks by UWB and Inertial Data Fusion," in arXiv:1305.1657. arXiv, 2013.

[20] V. Malyavej, W. Kumkeaw, and M. Waraponi, "Indoor Robot Localization by RSSI/IMU Sensor Fusion," in Proceedings of the 2013 10th International Conference on Electrical Engineering/Electronics, Computer, Telecommunications and Information Technology, ECTI-CON 2013. IEEE, 2013.

[21] J. Schmid, T. Gdeke, W. Stork, and K. D. Mller-Glaser, "On the Fusion of Inertial Data for Signal Strength Localization," in Proceedings of the 8th Workshop on Positioning Navigation and Communication, WPNC 2011. IEEE, 2011.

[22] O. Woodman and R. Harle, "Pedestrian Localisation for Indoor Environments," in Proceedings of the 10th International Conference on Ubiquitous Computing, UbiComp '08. ACM, 2008, pp. 114-123.

[23] W. W.-L. Li, R. A. Iltis, and M. Z. Win, "A Smartphone Localization Algorithm Using RSSI and Inertial Sensor Measurement Fusion," in Proceedings of IEEE Global Telecomm. Conference, GLOBECOM 2013. IEEE, 2013.

[24] I. Borg and P. Groenen, Modern Multidimensional Scaling: Theory and Applications, ser. Springer Series in Statistics. Springer, 2005. [Online]. Available: http://books.google.it/books?id=duTODldZzRcC

[25] Z.-X. Chen, H.-W. Wei, Q. Wan, S.-F. Ye, and W.-L. Yang, "A supplement to multidimensional scaling framework for mobile location: A unified view," Signal Processing, IEEE Transactions on, vol. 57, no. 5, pp. 2030-2034, 2009.

[26] J. S. Seybold, Introduction to RF propagation. John Wiley \& Sons, 2005.

[27] Evidence Srl, "Mini flex board," 2014. [Online]. Available: http: //www.evidence.eu.com/products/flex113-flex-mini-kit.html

[28] IEEE 802.15.4 Std-2006, "Part 15.4: Wireless Medium Access Control (MAC) and Physical Layer (PHY) Specifications for Low-Rate Wireless Personal Area Networks (LR-WPANs),” June 2006. 